Article

\title{
Community Microgrid Scheduling Considering Network Operational Constraints and Building Thermal Dynamics
}

\author{
Guodong Liu ${ }^{1 *}$, Thomas B. Ollis ${ }^{1}$, Bailu Xiao ${ }^{1}$, Xiaohu Zhang ${ }^{2}$ and Kevin Tomsovic ${ }^{2}$, \\ 1 Power \& Energy Systems Group, Oak Ridge National Laboratory, Oak Ridge, TN 37831, USA; \\ liug@ornl.gov; ollistb@ornl.gov; xiaob@ornl.gov \\ 2 Department of Electrical Engineering and Computer Science, The University of Tennessee, Knoxville, TN \\ 37996, USA; xzhang46@utk.edu; tomsovic@utk.edu \\ * Correspondence: liug@ornl.gov; Tel.: +1-865-241-9732
}

\begin{abstract}
This paper proposes a Mixed Integer Conic Programming (MICP) model for community microgrids considering the network operational constraints and building thermal dynamics. The proposed multi-objective optimization model optimizes not only the operating cost, including fuel cost, purchasing cost, battery degradation cost, voluntary load shedding cost and the cost associated with customer discomfort due to room temperature deviation from the set point, but also several performance indices, including voltage deviation, network power loss and power factor at the Point of Common Coupling (PCC). In particular, the detailed thermal dynamic model of buildings is integrated into the distribution optimal power flow (D-OPF) model for the optimal operation. The heating, ventilation and air-conditioning (HVAC) systems can be scheduled intelligently to reduce the electricity cost while maintaining the indoor temperature in the comfort range set by customers. Numerical simulation results show the effectiveness of the proposed model and significant savings in electricity cost with network operational constraints satisfied.
\end{abstract}

Keywords: community microgrids; distribution optimal power flow; multiobjective optimization; thermal dynamic model; HVAC

\section{Introduction}

A microgrid can be defined as a low voltage distribution network comprising various distributed generation (DG), storage devices, and responsive loads that can be operated in both grid-connected and islanded modes [1]. It is connected to the main distribution network at the Point of Common Coupling (PCC), importing or exporting power to the distribution network as well as providing ancillary services, such as, voltage support, to the main distribution grid [2,3]. A microgrid can also improve local reliability, reduce emissions and contribute to lower cost of energy supply by taking advantage of the DG, storage devices and responsive loads [4]. Due to such benefits, the microgrid has attracted growing attention from both academia and industry [5].

The scheduling of microgrids in both grid-connected and islanded modes is usually performed by a microgrid controller. It determines the optimal dispatch of DG and the power exchanged between microgrid and distribution utility through PCC by solving a economic dispatch or unit commitment problem. The total operating cost is minimized subject to various technical, environmental, reliability and operating constraints. Considerable efforts have been devoted to optimal scheduling and management of microgrids [6]. Scheduling methods for a microgrid in islanded modes are proposed in [7]-[10]. In addition, numerous research and published works are devoted to the scheduling of microgrid in grid-connected mode [11]-[15]. In particular, deterministic models are used in [11] and [12], while stochastic programming models have been developed in [13]-[14]. A hybrid stochastic/robust programming model for microgrid scheduling is proposed in [15]. However, in most of existing literature, network configuration, reactive power flow and power factor constraints have been mostly ignored in the optimization model. Under this 
assumption, the voltage and power factor limits as well as line thermal limits may not be satisfied by the solution. For this reason, economic and secure operation of a microgrid requires solving a distribution optimal power flow (D-OPF) considering the distribution network, voltage and power factor limits, and so on. Various approaches have been proposed for solving the D-OPF problem. Generally, these approaches can be classified into three categories. In the first category, the nonlinear optimization problem is directly solved using nonlinear programming methods such as gradient search or interior point methods [16]-[18]. It should be noted that the solution time and convergence characteristic of nonlinear programming in solving the D-OPF problem may not satisfy the stringent time constraints required by real-time controls. More commonly, as in the second category, the nonlinear optimization problem is addressed by iteratively solving the linearized problem [19]-[23]. These linear programming based methods are generally more efficient in term of solution time. Nevertheless, the linearized problem is formulated based on the calculated sensitivity coefficients, which cannot represent the nonlinear system correctly when the solution is close to the optimum. As a result, the solution may oscillate around the optimum and fail to converge. In the third category, the non-linear/non-convex items in the D-OPF problems are directly approximated or relaxed into linear/convex form. Thus, the D-OPF is formulated as linear programming $[24,25]$; or semidefinite programming (SDP) [26]; or second-order cone programming (SOCP) using either polar coordinates [27] or the DistFlow model $[28,29]$ by several convex relaxations. The relaxed convex D-OPF preserves the accuracy of the power flow model while improving the solution efficiency, and thus, it has become more and more popular in recent years.

In the existing literature, HVAC consumption is mostly considered as a responsive load, which could be postponed, or as a simple non-controllable load. The thermal dynamic characteristics of buildings as well as the customer comfort preference are seldom taken into account. In fact, the slow thermal dynamic characteristic of buildings make HVAC systems perfect candidates for demand side management due to building thermal inertia. Given indoor temperature settings (desirable temperature and allowable temperature deviation) from customers, the HVAC system can precool (or preheat) the building by turning on during low price hours and turning off during high/peak price hours while still maintaining the indoor temperature in allowable range. By integrating the thermal dynamic model of buildings into the microgrid scheduling process and allowing the microgrid controller surrogate control of the HVAC systems, significant savings in electricity cost can be achieved while preserving comfort. Recently, optimal control schemes for HVAC considering thermal dynamic model of buildings has been proposed in [30]-[31]. Nevertheless, the HVAC system was modeled as a continuous controllable load, while in practical cases, most HVAC systems can only be switched on and off. In addition, the network configuration, voltage and power factor limits are neglected.

In this paper, we propose a new mixed integer conic programming (MICP) model for community microgrids considering the network operational constraints and building thermal dynamics. The proposed optimization framework optimizes not only the total operating cost, but also the performance indices, including voltage deviation, network power loss and power factor. Detailed thermal dynamic characteristics of buildings and HVAC system models are integrated into the proposed community microgrid scheduling model. The main contributions of this paper are as follows:

- proposing a multiobjective optimization model to optimize both the operating cost and performance indices of a microgrid. The weighting coefficients of each objective are determined using the analytical hierarchy process (AHP) [32].

- integrating the building thermal dynamics and HVAC systems controller into microgrid scheduling model and reducing operating cost of microgrids; and

- validating the proposed model with numerical simulation results.

The rest of this paper is organized as follows. In Section II, the model of community microgrid and thermal dynamic model of buildings are presented. Based on those models, the community microgrid 
scheduling model considering network operational constraints and building thermal dynamics is proposed in Section III. In Section IV, case studies and sensitivity analysis on a community microgrid are presented. Finally, conclusions are given in Section V.

\section{System Modeling}

\subsection{Community Microgrid Model}

In this paper, we consider a community microgrid that consists of various distributed generation (PV panels, microturbines, fuel cells, diesel generations, etc.), energy storage (e.g., batteries) and a number of houses. For each house, there are HVAC loads and non-HVAC loads. In addition, rooftop PV panels, small batteries and EVs may also be present. A building energy management system (BEMS) is equipped for each house to communicate with the microgrid controller. Generally, the objective of the microgrid controller is to minimize the total cost of operating the community microgrid by coordinating the DG and battery operation, customers' consumption and energy purchasing/selling at the PCC while preserving customers' comfort and satisfying operational constraints. In particular, we assume the microgrid controller collects customer indoor temperature settings (desirable temperature and allowable temperature deviation) and other required information from each BEMS. Based on the customer requirements, electricity demand, renewable generation and price information at the PCC, the microgrid controller decides the real and reactive power output of controllable DG, charging/discharging power of batteries, purchasing/selling power at PCC and power consumption of HVAC systems as well as other controllable loads over a scheduling period (e.g., 24 hours). An example of the community microgrid under consideration is shown in Figure 1.

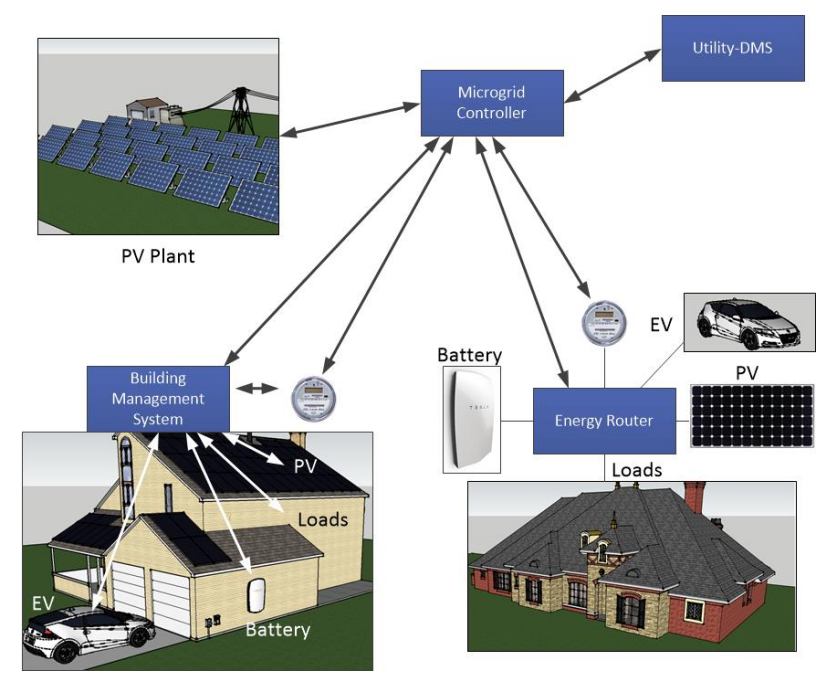

Figure 1. Example community microgrid

\subsection{HVAC System Model}

HVAC systems are typically controlled by thermostats to maintain the house indoor temperature in a comfortable range. Depending on customers' preference, the desirable indoor temperature and allowable temperature range will be preset. Inside the thermostats, a temperature controlled relay circuit is used to switch the HVAC on and off according to the temperature detected by the temperature sensor. The output from the thermostats is either on or off. For cooling control, the HVAC system is switched on when the indoor temperature reaches the ceiling of the allowed temperature range. It will be switched off when the indoor temperature falls below the floor of the allowed temperature range. This autonomous device has been widely used in buildings and houses where automatic temperature control is needed. 
Different from the autonomous temperature control above, we assume the desirable temperature and allowable temperature range set by customers are forwarded to the community microgrid controller by the BEMS and the microgrid controller takes surrogate control of HVAC systems. Since indoor temperature changes quite slowly due to thermal inertia, a house can be considered as a thermal storage facility. This gives the microgrid controller certain flexibility to schedule the consumption of HVAC system. Specially, a microgrid controller can switch on HVAC systems during low price or high renewable generation intervals to precool (or preheat) the house and switch off in opposite cases while still maintaining the indoor temperature in allowable range. As a result, it is expected that significant savings in electricity cost can be achieved compared to autonomous temperature control.

\subsection{Building Thermal Dynamic Model}

Thermal energy is transferred from a higher temperature space toward a lower temperature space due to conduction, convection, and radiation. Based on heat transfer mechanisms, we construct a third order linear model for the thermal dynamics of a house considering the impact of ambient temperature, solar irradiance and HVAC systems [33] as:

$$
\begin{gathered}
C_{h}^{\mathrm{M}} \frac{d T_{h}^{\mathrm{M}}}{d t}=\frac{1}{R_{h}^{\mathrm{M}}}\left(T_{h}^{\mathrm{In}}-T_{h}^{\mathrm{M}}\right)+A_{h} p_{h}^{s} \Phi_{h}^{s} \\
C_{h}^{\mathrm{E}} \frac{d T_{h}^{\mathrm{E}}}{d t}=\frac{1}{R_{h}^{\mathrm{E}}}\left(T_{h}^{\mathrm{In}}-T_{h}^{\mathrm{E}}\right)+\frac{1}{R_{h}^{\mathrm{EA}}}\left(T^{\mathrm{A}}-T_{h}^{\mathrm{E}}\right) \\
C_{h}^{\mathrm{In}} \frac{d T_{h}^{\mathrm{In}}}{d t}=\frac{1}{R_{h}^{\mathrm{A}}}\left(T^{\mathrm{A}}-T_{h}^{\mathrm{In}}\right)+\frac{1}{R_{h}^{\mathrm{M}}}\left(T_{h}^{\mathrm{M}}-T_{h}^{\mathrm{In}}\right) \\
+\frac{1}{R_{h}^{\mathrm{E}}}\left(T_{h}^{\mathrm{E}}-T_{h}^{\mathrm{In}}\right)+A_{h}\left(1-p_{h}^{s}\right) \Phi_{h}^{s}+\left(u_{h t}^{H}-u_{h t}^{C}\right) \eta_{h} P_{h}^{H}
\end{gathered}
$$

The equivalent thermal parameters $R_{h}^{\mathrm{A}}, R_{h}^{\mathrm{M}}, R_{h}^{\mathrm{E}}, R_{h}^{\mathrm{EA}}, C_{h}^{\mathrm{In}}, C_{h}^{\mathrm{M}}, C_{h}^{\mathrm{E}}$, and $p_{h}^{s}$ are assumed to be constant for a house $h$, which can be estimated by using various methods based on measured data $[34,35]$. Thus, the above thermal dynamics of house $h$ can be rewritten in the linear state-space model in continuous time as:

$$
\frac{d \boldsymbol{T}_{h}}{d t}=\boldsymbol{A}_{h} \boldsymbol{T}_{h}+\boldsymbol{B}_{h} \boldsymbol{U}_{h}
$$

where $\boldsymbol{T}_{h}=\left[T_{h}^{\mathrm{In}}, T_{h}^{M}, T_{h}^{E}\right]$ is the state vector. $\boldsymbol{U}_{h}=\left[T^{A}, \Phi,\left(u_{h}^{H}-u_{h}^{C}\right) \eta_{h} P_{h}^{H}\right]$ is the input vector. The coefficients of matrices $\boldsymbol{A}_{h}$ and $\boldsymbol{B}_{h}$ of a house $h$ can be calculated based on the effective window area, the fraction of solar irradiation entering the inner walls and floor, the thermal capacitance and resistance parameters of the house. The state-space model in continuous time (4) can be further transformed into equivalent discrete time model (5) by using Euler discretization (i. e., zero-order hold) with a sampling time [33,36], which is the time resolution of the optimization horizon

$$
\boldsymbol{T}_{h, t+1}=\boldsymbol{A}_{h}^{d} \boldsymbol{T}_{h, t}+\boldsymbol{B}_{h}^{d} \boldsymbol{U}_{h, t} \quad \forall h, \forall t
$$

Where $\boldsymbol{A}_{h}^{d}$ and $\boldsymbol{B}_{h}^{d}$ are constant, which can be calculated based on $\boldsymbol{A}_{h}, \boldsymbol{B}_{h}$ and the sampling time. This discrete time thermal dynamic model will be used in the following problem formulation. More details on this building thermal dynamic model can be found in [33]. The constraints on house indoor temperature are:

$$
T_{h t}^{D}-\delta_{h t} \leq T_{h t}^{\mathrm{In}} \leq T_{h t}^{D}+\delta_{h t}
$$




\section{Problem Formulation}

\subsection{Objective}

In the context of microgrids, dispatchable and undispatchable generation as well as batteries are integrated into the distribution network. The microgrid is connected to the external distribution system via the PCC characterized by a forecast day-ahead hourly cost of energy exchange known for a time window of 24 hours. Depending on unit type, dispatchable units are subject to various constraints, such as, capacity limits, minimum power output limits, ramping rates, minimum on/off time, and so on. In contrast, renewable generation, such as PV panels, are taken as non-dispatchable units, which depend on the meteorological conditions of ambient temperature and solar irradiance. Each house or building is associated with a HVAC load and a non-HVAC load. Under this assumption, the operation objective of a microgrid aims at minimizing a virtual cost associated with the system operating cost and performance as in (7). Specifically, the first and second line is the fuel cost of DGs (including DG start-up cost); the third line is the discomfort cost of customers due to the deviations between actual temperature and customer desired temperature and cost of load shedding; the fourth line is the energy purchasing/selling cost/benefit from distribution grid and cost of battery degradation; the fifth and sixth lines are total voltage deviation; and the seventh line is the total network loss and the total absolute value of reactive power exchange, which indicates the power factor at the PCC. These objectives are combined into a single objective function by a weighted summation.

$$
\begin{aligned}
\min \quad & W_{C}\left\{\sum_{t=1}^{N_{T}} \sum_{i=1}^{N_{G}}\left[\sum_{m=1}^{N_{I}} \lambda_{i t}(m) p_{i t}(m)+\kappa_{i} u_{i t}\right]\right. \\
& +\sum_{t=1}^{N_{T}} \sum_{i=1}^{N_{G}} S U_{i t}\left(u_{i t}, u_{i, t-1}\right) \\
& +\sum_{t=1}^{N_{T}} \sum_{h=1}^{N_{H}}\left(\omega_{h t}\left|T_{h t}^{\mathrm{In}}-T_{h t}^{\mathrm{D}}\right|+V_{h t}^{L S} P_{h t}^{L S}\right) \\
& \left.+\sum_{t=1}^{N_{T}} \lambda_{t}^{\mathrm{PCC}} P_{t}^{\mathrm{PCC}}+\sum_{t=1}^{N_{T}} \sum_{b=1}^{N_{B}} C_{b t}\left(P_{b t}^{\mathrm{C}}+P_{b t}^{\mathrm{D}}\right)\right\} \\
& +W_{V}\left[\sum_{t=1}^{N_{T}} \sum_{n=1}^{N_{N}} V_{n t}^{2}-\left(V_{\mathrm{thr}}^{\mathrm{max}}\right)^{2}:\left(V_{n t}>V_{\mathrm{thr}}^{\max }\right)\right. \\
& \left.+\sum_{t=1}^{N_{T}} \sum_{n=1}^{N_{N}}\left(V_{\mathrm{thr}}^{\min }\right)^{2}-V_{n t}^{2}:\left(V_{n t}<V_{\mathrm{thr}}^{\min }\right)\right] \\
& +W_{L}\left[\sum_{t=1}^{N_{T}} \sum_{j=1}^{N_{F}} r_{f} l_{f t}\right]+W_{Q}\left(\sum_{t=1}^{N_{T}}\left|Q_{t}^{\mathrm{PCC}}\right|\right)
\end{aligned}
$$

The weighting coefficients $W_{C}, W_{V}, W_{L}$ and $W_{Q}$ can be determined by using the analytical hierarchy process (AHP) [32]. First of all, a pairwise comparison is done between the objectives. The relative importance of each factor in comparison with all other factors will be identified by the decision-maker, who may have difference preferences from network to network. Then, a matrix is built based on these comparisons. Finally, the weighting coefficients are calculated based on the matrix. 
6 of 20

\subsection{Constraints}

The objective function must be minimized subject to a number of constraints. For simplicity, these constraints are grouped into DG constraints, battery constraints, load constraints, network constraints and constraints at PCC.

\subsubsection{DG Constraints}

The constraints associated with DGs include the following:

$$
\begin{gathered}
P_{i t}=\sum_{m=1}^{N_{I}} p_{i t}(m)+u_{i t} P_{i}^{\min } \quad \forall i, \forall t \\
0 \leq p_{i t}(m) \leq p_{i t}^{\max }(m) \quad \forall i, \forall t, \forall m \\
P_{i t} \leq P_{i}^{\max } u_{i t} \quad \forall i, \forall t \\
-\tan \left(\theta_{i}\right) P_{i t} \leq Q_{i t} \leq \tan \left(\theta_{i}\right) P_{i t} \quad \forall i, \forall t \\
\left(P_{i t}\right)^{2}+\left(Q_{i t}\right)^{2} \leq S_{i}^{2} \quad \forall i, \forall t
\end{gathered}
$$

Constraints (8) and (9) approximate the fuel cost of DGs by blocks. Constraint (10) enforces the output of DG to be zero if it is not committed. The power factor and capacity limit is ensured by (11) and (12).

\subsubsection{Battery Constraints}

The constraints associated with batteries include the following:

$$
\begin{gathered}
0 \leq P_{b t}^{\mathrm{C}} \leq P_{b}^{\mathrm{C}, \max } u_{b t}^{\mathrm{C}} \quad \forall b, \forall t \\
0 \leq P_{b t}^{\mathrm{D}} \leq P_{b}^{\mathrm{D}, \max } u_{b t}^{\mathrm{D}} \quad \forall b, \forall t \\
u_{b t}^{\mathrm{C}}+u_{b t}^{\mathrm{D}} \leq 1 \quad \forall b, \forall t \\
S O C_{b t}=S O C_{b, t-1}+P_{b t}^{\mathrm{C}} \eta_{b}^{\mathrm{C}} \triangle t-P_{b t}^{\mathrm{D}} \frac{1}{\eta_{b}^{\mathrm{D}}} \Delta t \quad \forall b, \forall t \\
S O C_{b t}^{\min } \leq S O C_{b t} \leq S O C_{b t}^{\max } \quad \forall b, \forall t \\
-\tan \left(\theta_{b}^{\mathrm{C}}\right) P_{b t}^{\mathrm{C}} \leq Q_{b t} \leq \tan \left(\theta_{b}^{\mathrm{C}}\right) P_{b t}^{\mathrm{C}}:\left(P_{b t}^{\mathrm{C}}>0\right) \forall b, \forall t \\
-\tan \left(\theta_{b}^{\mathrm{D}}\right) P_{b t}^{\mathrm{D}} \leq Q_{b t} \leq \tan \left(\theta_{b}^{\mathrm{D}}\right) P_{b t}^{\mathrm{D}}:\left(P_{b t}^{\mathrm{D}}>0\right) \forall b, \forall t \\
P_{b t}=P_{b t}^{\mathrm{D}}-P_{b t}^{\mathrm{C}} \forall b, \forall t \\
\left(P_{b t}\right)^{2}+\left(Q_{b t}\right)^{2} \leq S_{b}^{2} \quad \forall b, \forall t
\end{gathered}
$$


Constraint (13) and (14) are the maximum charging/discharging power of a battery. These two states are mutually exclusive, which is ensured by (15). The state of charge (SOC) limits are enforced by (16) and (17). In (16), the battery SOC at the end of current time interval equals to the SOC at the end of last time interval plus the energy charged minus the energy discharged during current interval. The charging and discharging of battery is subject to loss, which is represented by parameter $\eta_{b}^{C}$ and $\eta_{b}^{\mathrm{D}}$. The power factor limits of a battery in both charging and discharging states are represented by (18) and (19). The capacity limit is ensured by (20) and (21). The logical constraints (18) - (19) will be simplified into mixed integer linear (MIL) format in subsection 3.3.

\subsubsection{Load Constraints}

For each house, the load is divided into HVAC load and non-HVAC load as in equation (22) and (23). The constraints associated with HVAC systems include equation (5) and (6). Since the heating and cooling states of a HVAC system are mutually exclusive, this is ensured by (24). The amount of load curtailment in house $h$ at time $t$ is limited by constraint (25) and (26).

$$
\begin{gathered}
P_{h t}=\left(u_{h t}^{\mathrm{H}}+u_{h t}^{\mathrm{C}}\right) P_{h}^{\mathrm{H}}+P_{h t}^{\mathrm{O}} \quad \forall h, \forall t \\
Q_{h t}=\tan \left(\varphi_{h}^{\mathrm{H}}\right)\left(u_{h t}^{\mathrm{H}}+u_{h t}^{\mathrm{C}}\right) P_{h}^{\mathrm{H}}+Q_{h t}^{\mathrm{O}} \quad \forall h, \forall t \\
u_{h t}^{\mathrm{H}}+u_{h t}^{\mathrm{C}} \leq 1 \quad \forall h, \forall t \\
0 \leq P_{h t}^{\mathrm{LS}} \leq P_{h t}^{\mathrm{LS}, \max } \quad \forall h, \forall t \\
Q_{h t}^{\mathrm{LS}}=\tan \left(\varphi_{h}^{\mathrm{O}}\right) P_{h t}^{\mathrm{LS}} \quad \forall h, \forall t
\end{gathered}
$$

\subsubsection{Network Constraints}

The model of a distribution feeder with bus $n$ as sending end and bus $n+1$ as receiving end is shown in Figure 2. Considering the power flow direction as depicted in Figure 2. Equation (27) (30) represents the DistFlow equations, which have been verified in $[26,29]$. The nodal voltage drop along a feeder is represented by (27). The real and reactive power balances across the network are enforced by (28) and (29), where $\mathbf{M}_{\mathbf{f}}$ and $\mathbf{M}_{\mathbf{l}}$ are the incidence matrix for the sending end line flow and line losses. $\mathbf{M}_{\mathbf{f}}(n, f)$ is 1 if bus $n$ is the sending end of line $f$, and -1 if bus $n$ is the receiving end of line $f$ and zero if neither terminal of line $f$ is connected to bus $n . \mathbf{M}_{\mathbf{l}}(n, f)$ is 1 if bus $n$ is the receiving end of line $f$ and zero for all other elements. It should be noted that $P_{t}^{\mathrm{PCC}}$ and $Q_{t}^{\mathrm{PCC}}$ should be added to (28) and (29), separately, for the PCC node. The quadratic equality constraint in (30) is nonconvex, but we can relax this into inequalities as in (31) by conic relaxation. For the accuracy of the conic relaxation in optimal power flow (OPF) problem, we refer readers to [37,38], which compare the conic relaxation based OPF results with standard nonlinear programming (NLP) based OPF results for different IEEE test systems. Equation (31) is reformulated as a standard second order cone formulation in (32), which will be used in the problem formulation instead of (30) and (31). The maximum voltage deviation of all nodes is represented by (33) and the line thermal limits are represented by (34). Note that the square of nodal voltages in (27) and (33) is directly taken as a variable. Thus, these two constraints are linear. 


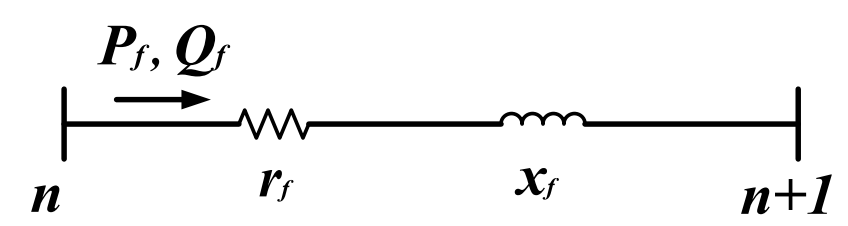

Figure 2. Model of distribution feeder

$$
\begin{aligned}
& V_{n+1, t}^{2}=V_{n t}^{2}-2\left(r_{f} P_{f t}+x_{f} Q_{f t}\right)+\left(r_{f}^{2}+x_{f}^{2}\right) l_{f t} \quad \forall f, \forall t \\
& \sum_{i \in \mathcal{G}_{n}} P_{i t}+\sum_{b \in \mathcal{B}_{n}} P_{b t}-\sum_{h \in \mathcal{H}_{n}} P_{h t}+\sum_{h \in \mathcal{H}_{n}} P_{h t}^{\mathrm{LS}}+\sum_{v \in \mathcal{V}_{n}} P_{v t}=\sum_{f \in \Omega} \mathbf{M}_{\mathbf{f}}(n, f) P_{f t}+\sum_{f \in \Omega} \mathbf{M}_{\mathbf{l}}(n, f) r_{f} l_{f t} \quad \forall n, \forall t \\
& \sum_{i \in \mathcal{G}_{n}} Q_{i t}+\sum_{b \in \mathcal{B}_{n}} Q_{b t}-\sum_{h \in \mathcal{H}_{n}} Q_{h t}+\sum_{h \in \mathcal{H}_{n}} Q_{h t}^{\mathrm{LS}}+\sum_{v \in \mathcal{V}_{n}} Q_{v t}=\sum_{f \in \Omega} \mathbf{M}_{\mathbf{f}}(n, f) Q_{f t}+\sum_{f \in \Omega} \mathbf{M}_{\mathbf{l}}(n, f) x_{f} l_{f t} \quad \forall n, \forall t \\
& \left(P_{f t}\right)^{2}+\left(Q_{f t}\right)^{2}=V_{n t}^{2} l_{f t} \quad \forall f, \forall t \\
& \left(P_{f t}\right)^{2}+\left(Q_{f t}\right)^{2} \leq V_{n t}^{2} l_{f t} \quad \forall f, \forall t \\
& \left\|\begin{array}{c}
2 P_{f t} \\
2 Q_{f t} \\
l_{f t}-V_{n t}^{2}
\end{array}\right\|_{2} \leq l_{f t}+V_{n t}^{2} \quad \forall f, \forall t \\
& \left(V^{\min }\right)^{2} \leq V_{n t}^{2} \leq\left(V^{\max }\right)^{2} \quad \forall n, \forall t \\
& l_{f t} \leq\left(I_{f}^{\max }\right)^{2} \quad \forall f, \forall t
\end{aligned}
$$

\subsubsection{Constraints at PCC}

At the PCC, the exchanged power at PCC is subject to a maximum value due to line capacity or reliability requirements as in (35). The power factor limit at PCC is ensured by (36). In addition, the PCC voltage is normally determined by the utility network operating condition, which is specified in (37) .

$$
\begin{gathered}
-P^{\mathrm{PCC}, \mathrm{max}} \leq P_{t}^{\mathrm{PCC}} \leq P^{\mathrm{PCC}, \max } \quad \forall t \\
-\tan \left(\theta^{\mathrm{PCC}, \max }\right)\left|P_{t}^{\mathrm{PCC}}\right| \leq Q_{t}^{\mathrm{PCC}} \leq \tan \left(\theta^{\mathrm{PCC}, \max }\right)\left|P_{t}^{\mathrm{PCC}}\right| \\
V_{t}^{\mathrm{PCC}}=V^{\mathrm{Fix}}
\end{gathered}
$$

\subsection{Simplification and Linearization}

To reformulate the D-OPF problem into a MICP, all logical terms that appear in the objective function and constraints will be recast into MIL form. For the objective function, the startup cost of 
DGs (line 2) can be recast into mixed-integer linear form as in [39]. The logic expression of voltage deviation (line 5 and 6) can be reformulated into linear format as in (38) - (40) by introducing auxiliary variable $X_{n t}^{V}$, which represents the voltage deviation. Similarly, the expression of customer discomfort cost (line 3) can be reformulated into linear format as in (41) - (43) by introducing an auxiliary variable $X_{h t}$, which represents the absolute value of temperature deviation. The same technique can linearize other absolution functions in equation (7) and (36) into linear format. Thus, the proposed optimization becomes a MILP, which can be solved efficiently by commercial solvers.

$$
\begin{gathered}
X_{n t}^{V} \geq V_{n t}^{2}-\left(V_{\mathrm{thr}}^{\max }\right)^{2} \quad \forall n, \forall t \\
X_{n t}^{V} \geq\left(V_{\mathrm{thr}}^{\mathrm{min}}\right)^{2}-V_{n t}^{2} \quad \forall n, \forall t \\
X_{n t}^{V} \geq 0 \quad \forall n, \forall t \\
X_{h t} \geq T_{h t}^{\mathrm{In}}-T_{h t}^{D} \quad \forall h, \forall t \\
X_{h t} \geq T_{h t}^{D}-T_{h t}^{\mathrm{In}} \quad \forall h, \forall t \\
X_{h t} \geq 0 \quad \forall h, \forall t
\end{gathered}
$$

The logical terms in battery constraints (18) and (19) can be reformulated into MIL form, as (44) and (45) by the reuse of binary charging/discharging indicators

$$
\begin{aligned}
& -S_{b} u_{b t}^{\mathrm{D}}-\tan \left(\theta_{b}^{\mathrm{C}}\right) P_{b t}^{\mathrm{C}} \leq Q_{b t} \leq \tan \left(\theta_{b}^{\mathrm{C}}\right) P_{b t}^{\mathrm{C}}+S_{b} u_{b t}^{\mathrm{D}} \forall b, \forall t \\
& -S_{b} u_{b t}^{\mathrm{C}}-\tan \left(\theta_{b}^{\mathrm{D}}\right) P_{b t}^{\mathrm{D}} \leq Q_{b t} \leq \tan \left(\theta_{b}^{\mathrm{D}}\right) P_{b t}^{\mathrm{D}}+S_{b} u_{b t}^{\mathrm{C}} \forall b, \forall t
\end{aligned}
$$

Finally, the objective function has been reformulated into MIL form and all constraints are either MIL or second order cones (SOC) . Thus, the proposed D-OPF problem is represented as a MICP, which can be solved by commercial solvers efficiently.

\section{Case Studies}

The proposed community microgrid scheduling model is demonstrated on a modified ORNL Distributed Energy Control and Communication (DECC) lab microgrid test system as shown in Figure 3. The modified system includes various DERs, including PV panel, fuel cell, microturbine, diesel generator and battery. The parameters for the dispatchable generators, PV, wind turbine and battery can be found in [15]. The three dispatchable generators are duplicated to make sure the microgrid can be operated in islanded mode without load shedding. The solar irradiance and temperature data is from [40]. The measured 1-minute data of Oak Ridge, Tennessee area on August 1st, 2015 is used for the simulation. This is a typical summer day in southern states of the US. The cost of load shedding is set as $\$ 2.0 / \mathrm{kWh}$ and the amount of curtailed load should be less than $10 \%$ of the corresponding non-HVAC load in each house. Twenty houses are considered, each has a $5 \mathrm{~kW}$ HVAC system, which has a coefficient of performance (COP) $\eta_{h}=3$. The desired indoor temperature is set at $23{ }^{\circ} \mathrm{C}$ and the allowed deviation is $\pm 2{ }^{\circ} \mathrm{C}$. The customer discomfort factor is set at $\$ 0.05 /{ }^{\circ} \mathrm{C}$. All other parameters of the houses are taken from Table 7.1 in [33]. To represent the variety of the houses, the standard error of estimate in [33] (again Table 7.1) is used to generate random errors for the parameters of each house. This microgrid system includes various generation sources, such as, PV panel, fuel cell, microturbine and diesel generator as well as energy storage devices, such as, 
a battery. On the demand side, it considers multiple houses with different parameters. Thus, the modified DECC lab microgrid system covers a wide variety of both existing and future community microgrids components.

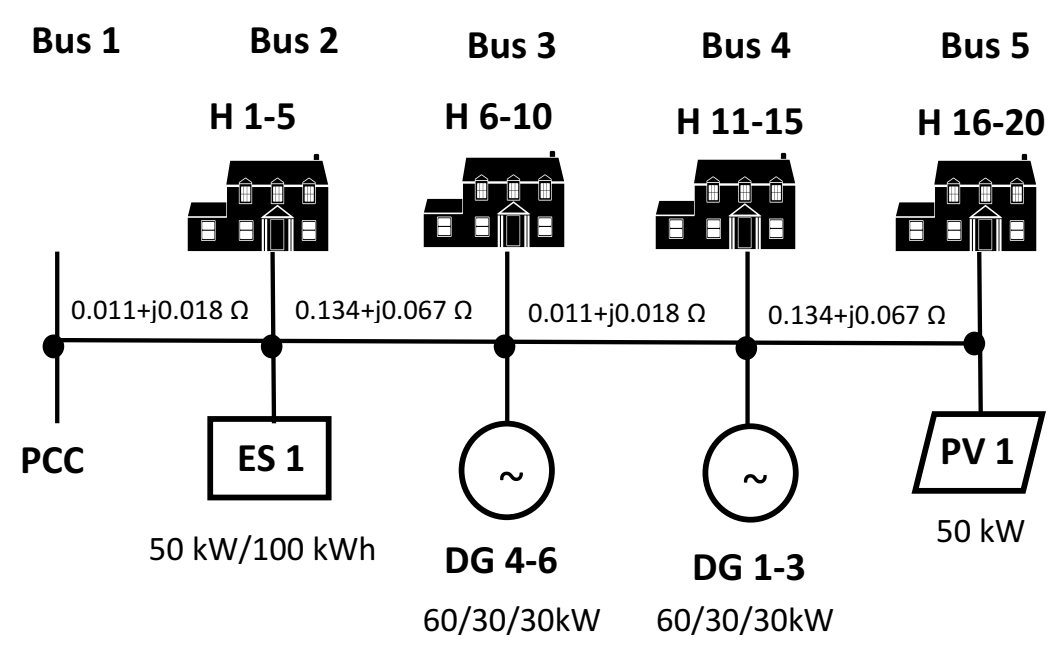

Figure 3. Community microgrid test system

The analysis is conducted for a 24-hour scheduling horizon and the time interval is set to be 15 minutes. The PV model is the same as in [18]. The forecast non-HVAC demand in house \#1 and the day-ahead market prices at the PCC are shown in Figure 4. The non-HVAC load curves of other houses are assumed the same. The power factor of HVAC is assumed 0.9 and the power factor at PCC is required to be no less than 0.9. All numerical simulations are coded in MATLAB and solved using the MICP solver CPLEX 12.6. With a pre-specified duality gap of $0.5 \%$, the running time is about 1 minute on a $2.66 \mathrm{GHz}$ Windows-based PC with $4 \mathrm{G}$ bytes of RAM.

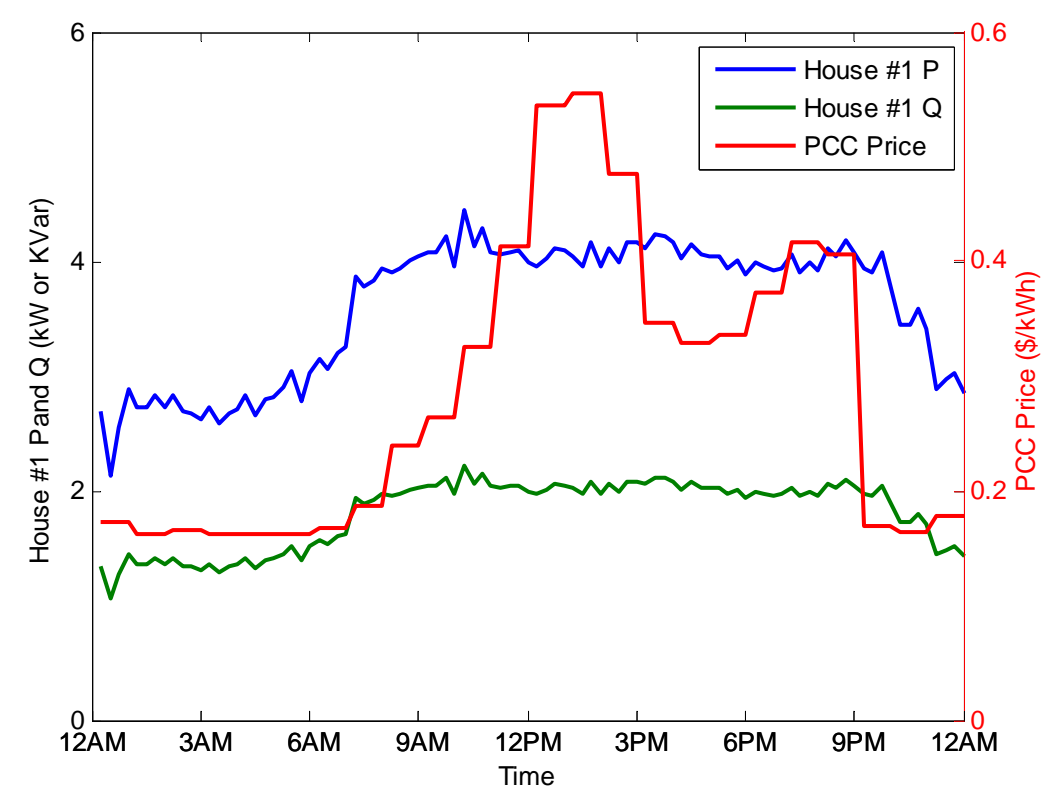

Figure 4. Non-HVAC demand in house and electricity price at the PCC 


\subsection{Effect of Weighting Factors}

As mentioned above, the weighting coefficients of each term in the objective function are determined by using the AHP. For simplicity, fuel cost, purchasing cost, battery degradation cost, load shedding cost and customers' discomfort cost are aggregated together as total cost with weighting coefficient $W_{C}$. First of all, a pairwise comparison of relative importance or priority between each two objectives is done. A pairwise comparison matrix can be established as in Table 1. For each position in the matrix, the importance of the objective at the left is compared with that of the objective at the top and the ratio is entered. Then, the AHP retrieves the weighting coefficients of all objectives by solving a trivial eigenvalue problem. If the pairwise comparison matrix is consistent, it is rank one and thus all its eigenvalues but one are equal to zero. This largest eigenvalue, i.e., principal eigenvalue is unique and equals to the dimension of the matrix. The corresponding eigenvector, i.e., principal eigenvector is positive and unique. The weighting coefficients of objectives can be obtained by normalizing this principal eigenvector. Since each element in the pairwise comparison matrix is directly estimated by comparing the priority of two corresponding objectives, the pairwise comparison matrix is very unlikely to be consistent, especially when the number of objective is large. According to [32], a consistency of about $10 \%$ is considered acceptable. In the inconsistent case, the principal eigenvalue is still unique and near the dimension of the matrix. The weighting coefficients of objectives can be obtained in the same way.

Based on the pairwise comparison matrix in Table 1, the weighting coefficients of each term are calculated as: $W_{C}=0.2084, W_{Q}=0.1171, W_{V}=0.6116$, and $W_{L}=0.0629$. It should be noted that the system operator can change the pairwise comparison matrix according to their system configuration and preference, and then the calculated weighting coefficients will be changed, accordingly.

Table 1. Pairwise comparisons of the objective terms

\begin{tabular}{c|c|c|c|c}
\hline & $\begin{array}{c}\text { Total } \\
\text { Cost }\end{array}$ & $\begin{array}{c}\text { Total } \\
\text { Reactive } \\
\text { Power }\end{array}$ & $\begin{array}{c}\text { Voltage } \\
\text { Deviation }\end{array}$ & $\begin{array}{c}\text { Network } \\
\text { Loss }\end{array}$ \\
\hline Total Cost & 1 & 2 & $1 / 3$ & 3 \\
\hline Total Reactive Power & $1 / 2$ & 1 & $1 / 5$ & 2 \\
\hline Voltage Deviation & 3 & 5 & 1 & 10 \\
\hline Network Loss & $1 / 3$ & $1 / 2$ & $1 / 10$ & 1 \\
\hline
\end{tabular}

Table 2. Evaluation of solutions of single objective optimization and multiobjective optimization for grid-connected operation

\begin{tabular}{|c|c|c|c|c|c|c|}
\hline & \multicolumn{4}{|c|}{ Single Objective Optimization } & \multirow{2}{*}{$\begin{array}{l}\text { Multiobjective } \\
\text { Optimization }\end{array}$} & \multirow{2}{*}{$\begin{array}{l}\text { Autonomous } \\
\text { Control }\end{array}$} \\
\hline & $\begin{array}{l}\text { Optimizing } \\
\text { Cost }\end{array}$ & $\begin{array}{l}\text { Optimizing } \\
\text { Loss }\end{array}$ & $\begin{array}{l}\text { Optimizing } \\
\text { Voltage }\end{array}$ & $\begin{array}{c}\text { Optimizing } \\
\text { Reactive } \\
\text { Power }\end{array}$ & & \\
\hline $\begin{array}{c}\text { Total Cost } \\
(\$)\end{array}$ & 521.49 & 661.38 & 558.65 & 584.27 & 531.73 & 634.75 \\
\hline $\begin{array}{c}\text { Loss } \\
(\mathrm{kWh})\end{array}$ & 51.95 & 5.8197 & 14.6587 & 35.8221 & 25.77 & 23.59 \\
\hline $\begin{array}{c}\text { Voltage } \\
\text { Deviation } \\
(\mathrm{pu}) \\
\end{array}$ & 6.12 & 0.24 & 0.016 & 11.63 & 2.06 & 1.45 \\
\hline $\begin{array}{l}\text { Reactive } \\
\text { Power } \\
\text { (KVARh) }\end{array}$ & 242.14 & 0.11 & 0.05 & 0 & 0.06 & 0.04 \\
\hline
\end{tabular}


In order to show the benefit of multiobjective optimization, both single objective optimization problems and a multiobjective optimization problem are solved for grid-connection operation. The solutions, i.e. real and reactive power of DGs and battery are evaluated by calculating the corresponding terms, i.e., total cost, network loss, voltage deviation and total reactive power at PCC. The results are compared in Table 2. When the problem is formulated as single objective optimization, the targeted term is always optimized to be the best (as shown in red) compared to that of other solutions (the rest in the same row). By the multiobjective optimization, none of these terms are always the best, but the objectives are optimized together. Network loss is weighted the least based on the AHP process. Nevertheless, all of these solutions are Pareto solutions, i.e., one cannot improve one term without sacrificing another.

\subsection{Comparison of Cost between Different Cases}

In order to show the benefit of integrating building thermal dynamics into community microgrid scheduling, we compare the total cost calculated by the proposed optimization and that of autonomous temperature control. In the case of autonomous control, the on/off state of HVAC system can be directly determined by solving equation (5) and performing the logic of temperature controlled relay. Then, the total cost can be calculated by scheduling the same microgrid with predetermined HVAC status. The weighting coefficients are set as: $W_{C}=0.2084, W_{Q}=0.1171, W_{V}=0.6116$, and $W_{L}=0.0629$ for all cases. Four cases are studied:

- Case 1: Autonomous temperature control in grid-connected mode

- Case 2: Proposed optimization in grid-connected mode

- Case 3: Autonomous temperature control in islanded mode

- Case 4: Proposed optimization in islanded mode

Table 3. Costs of the community microgrid in different cases

\begin{tabular}{c|c|c|c|c|c|c}
\hline \multicolumn{2}{c|}{ Cases } & $\begin{array}{c}\text { Total } \\
\text { Objective }\end{array}$ & $\begin{array}{c}\text { Operating } \\
\text { Cost }(\$)\end{array}$ & $\begin{array}{c}\text { Loss } \\
(\mathrm{kWh})\end{array}$ & $\begin{array}{c}\text { Voltage } \\
\text { Deviation } \\
(\mathrm{pu})\end{array}$ & $\begin{array}{c}\text { Reactive } \\
\text { Power } \\
(\mathrm{kVARh})\end{array}$ \\
\hline \multirow{2}{*}{ Grid-connected } & $\begin{array}{c}\text { Case 1: } \\
\text { Autonomous } \\
\text { Control }\end{array}$ & 134.66 & 634.75 & 23.59 & 1.45 & 0.04 \\
\cline { 2 - 7 } & $\begin{array}{c}\text { Case 2: } \\
\text { Proposed } \\
\text { Optimization }\end{array}$ & 113.70 & 531.73 & 25.77 & 2.06 & 0.06 \\
\hline $\begin{array}{c}\text { Case 3: } \\
\text { Autonomous } \\
\text { Control }\end{array}$ & 148.60 & 693.62 & 24.99 & 3.00 & 5.49 \\
\cline { 2 - 7 } & $\begin{array}{c}\text { Case 4: } \\
\text { Proposed } \\
\text { Optimization }\end{array}$ & 128.64 & 601.06 & 20.42 & 2.30 & 5.86 \\
\hline
\end{tabular}

The total objective as well as each objective values are compared in Table 3. As can be seen in grid-connected mode, the operating cost is reduced by $16.23 \%$ through the proposed optimization, while the system performance indices are almost the same. Similarly, in islanded mode, the operating cost is reduced by $13.34 \%$ through the proposed optimization without compromising the system performance. This clearly indicates significant cost savings can be achieved by the proposed microgrid scheduling model considering building thermal dynamics and network operational constraints. 


\subsection{Operation in Grid-Connected Mode}

The results of community microgrid scheduling in grid-connected mode through different control and optimization methods are compared. Taking house \#1 for example, the indoor temperature and HVAC status are shown in Figure 5. Comparing Figure 5a with Figure 5b clearly shows how the proposed model considering can precool the house to reduce the HVAC consumption during peak price intervals. In specific, by the proposed optimization, the HVAC in house \#1 is turned on before $9 \mathrm{AM}$ when the indoor temperature is still comfortable. The house is precooled, so the HVAC can be turned off during the pick price intervals between 1 PM-2 PM.
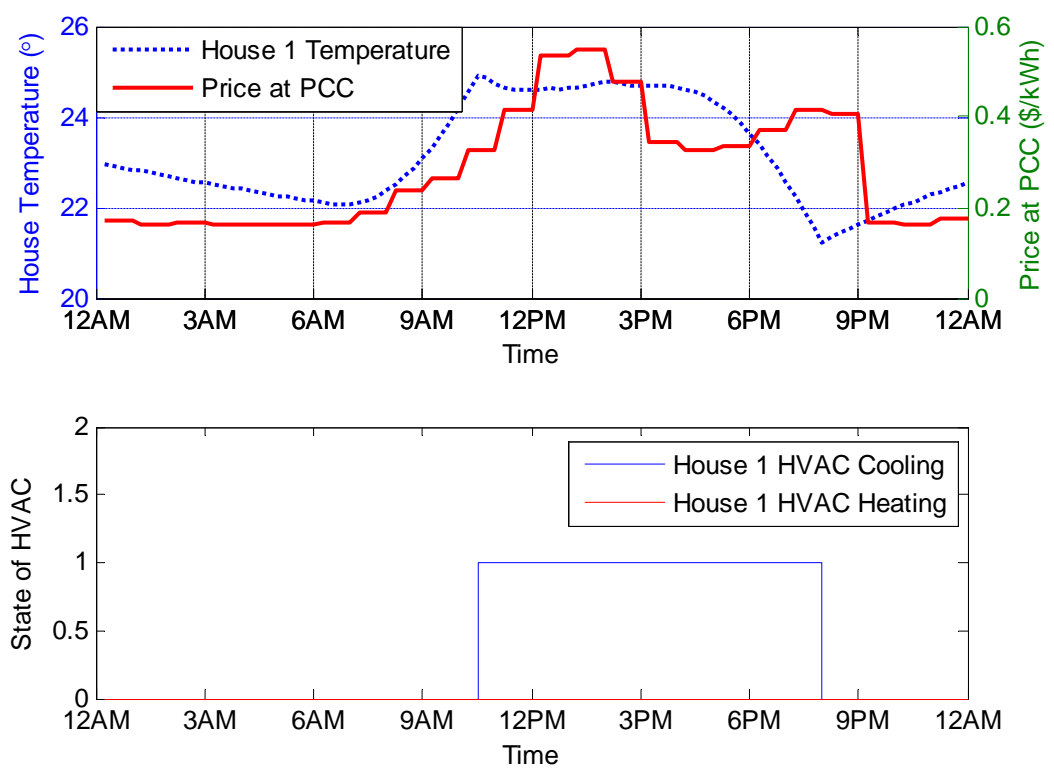

(a) Autonomous temperature control
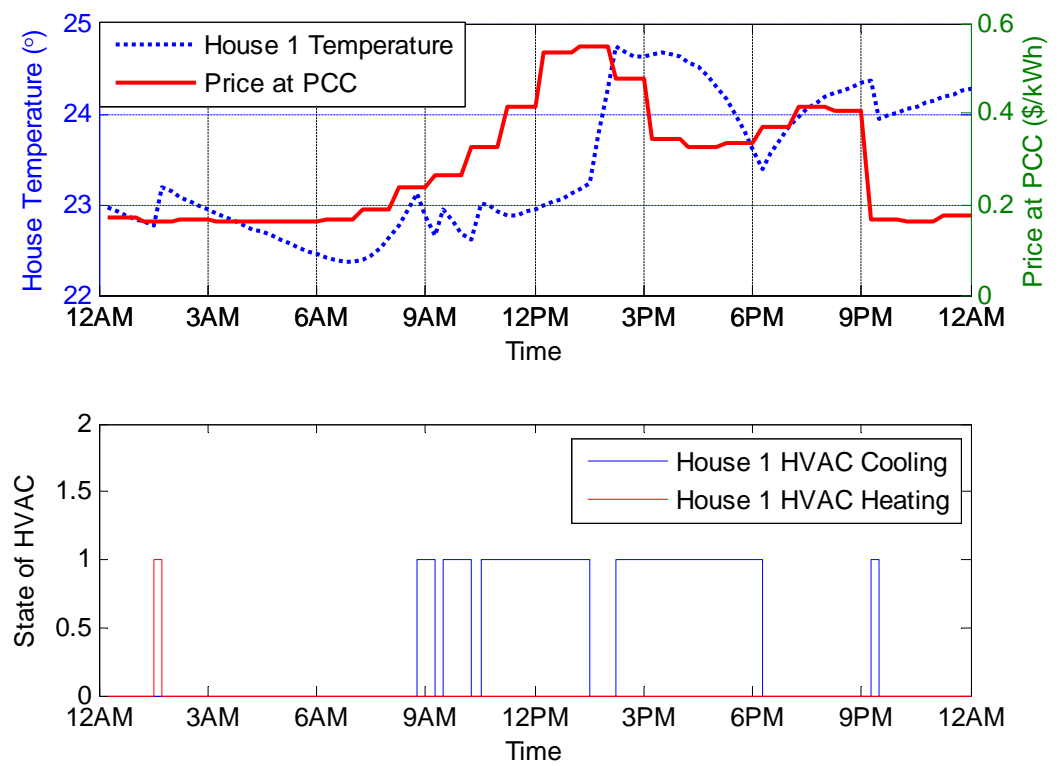

(b) Proposed Optimization

Figure 5. House temperature and HVAC status in grid-connected mode 
Figure 6 illustrates how the load is shifted by the proposed optimization comparing with the autonomous control method. As can be expected, the loads are shifted earlier in response to the price. Specifically, loads are shifted before the first price peak, so the second price peak can be avoided. With better thermal insulation of the house, the first price peak can also be avoided.
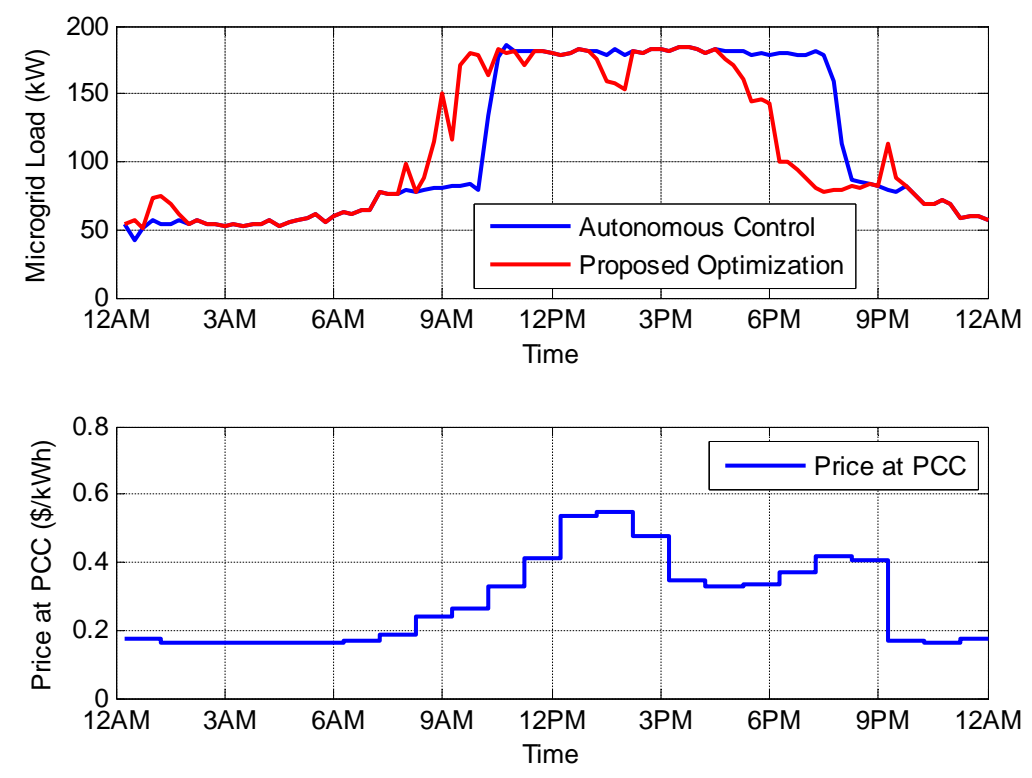

Figure 6. Load shifting in grid-connected mode

\subsection{Operation in Islanded Mode}

The results of community microgrid scheduling in islanded mode through different control and optimization methods are compared in Figure 7. In islanded model, the microgrid net demand, i.e., the total load (both HVAC and non-HVAC) minus the renewable generation and shed load is found. The net demand is a good indicator of the energy price. With more net demand, units with higher marginal cost need to be committed. Thus, the energy price will be higher. As can been seen in Figure $7 \mathrm{a}$ and Figure $7 \mathrm{~b}$, the peak net demand of the proposed optimization model considering building thermal dynamics has been significantly reduced compared to that of autonomous control in islanded mode. This indicates the peak energy prices of proposed optimization model are reduced compared to autonomous control. Similar to the grid-connected cases, the proposed model can precool the house to reduce the HVAC consumption during peak price/demand intervals. 

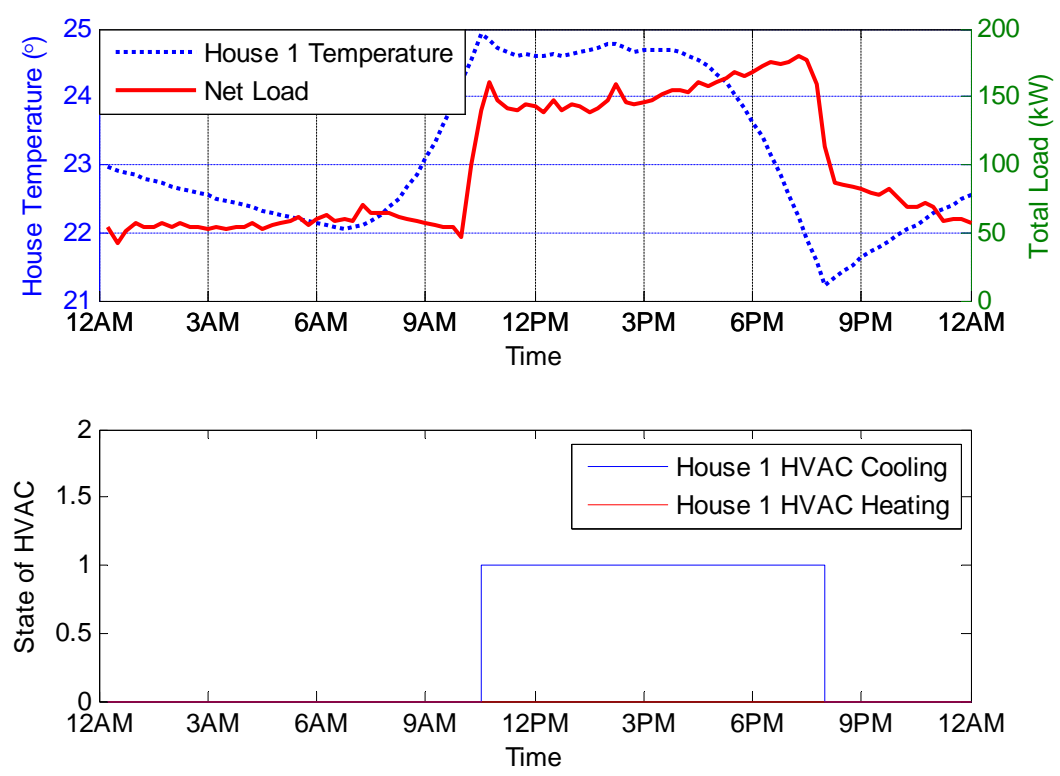

(a) Autonomous temperature control
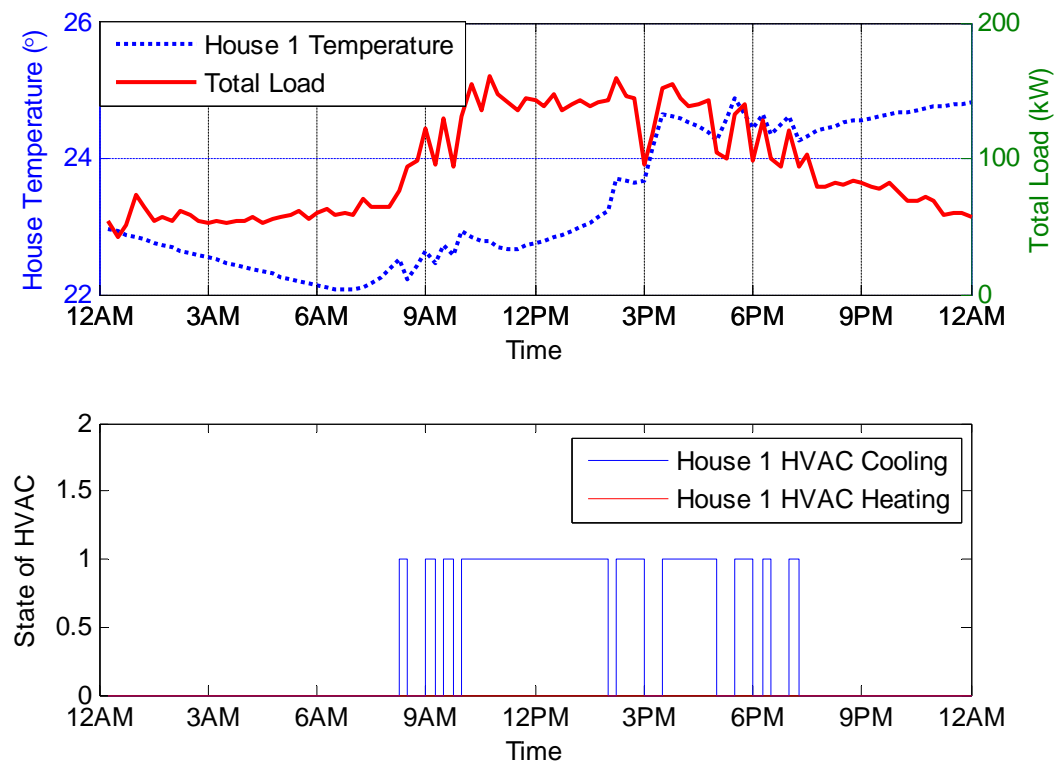

(b) Proposed Optimization

Figure 7. House temperature and HVAC status in islanded mode

Figure 8 illustrates how load is shifted in islanded mode. As expected, the loads are shifted earlier to reduce both the value and duration of the peak load. In this way, units with higher marginal cost will be not committed or committed for a shorter time. Thus, the total operating cost will be reduced. 


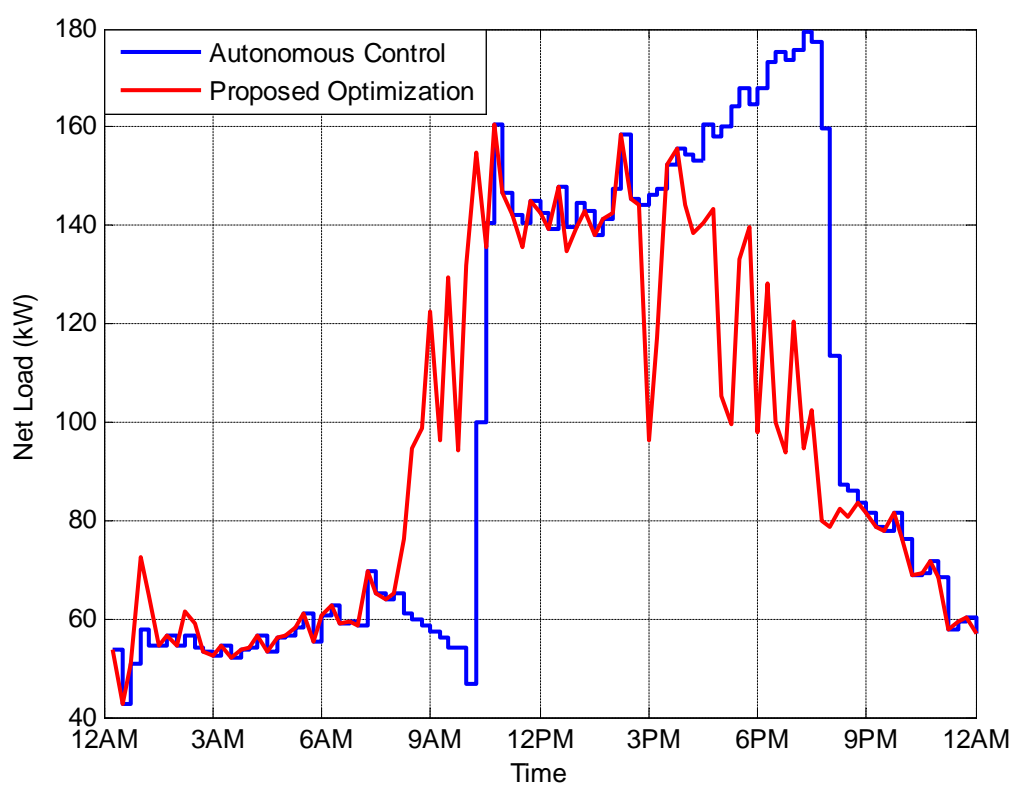

Figure 8. Load shifting in islanded mode

\section{Conclusions}

In this paper, an MICP-based community microgrid scheduling model considering building thermal dynamics and network operational constraints is proposed. The model directly integrates the detailed thermal dynamic characteristics of buildings into the optimization scheme and enables smart scheduling of HVAC systems. The proposed model optimizes both microgrid operating cost and system performance indices, such as network loss, voltage deviation and power factor at PCC. Numerical simulations on the modified ORNL DECC microgrid show the effectiveness of the proposed model and significant saving in electricity cost can be achieved with network operational constraints satisfied.

Acknowledgments: This work is supported by the U.S. Department of Energy's Office of Electricity Delivery and Energy Reliability (OE) under Contract No. DE-AC05-00OR22725. This work also made use of Engineering Research Center Shared Facilities supported by the Engineering Research Center Program of the National Science Foundation and the Department of Energy under NSF Award Number EEC-1041877 and the CURENT Industry Partnership Program.

Author Contributions: Guodong Liu carried out the main research tasks and wrote the full manuscript. Thomas B. Ollis and Bailu Xiao participated in the development of the proposed methodology and the literature review. Xiaohu Zhang and Kevin Tomsovic provided important suggestions on writing of the paper.

The authors declare no conflict of interest.

\section{Nomenclature}

The main symbols used in this paper are defined below. Others will be defined as required in the text. A bold symbol stands for its corresponding vector or matrix.

Indices and Sets

$i \quad$ Index of DGs, running from 1 to $N_{G}$.

$b \quad$ Index of batteries, running from 1 to $N_{B}$.

$h \quad$ Index of houses, running from 1 to $N_{H}$.

$v \quad$ Index of PV generation, running from 1 to $N_{V}$.

$f \quad$ Index of feeders, running from 1 to $N_{F}$.

$n \quad$ Index of buses, running from 1 to $N_{N}$. 
$t \quad$ Index of time periods, running from 1 to $N_{T}$.

$m$ Index of energy blocks offered by DGs, running from 1 to $N_{I}$.

$\mathcal{G}_{n} \quad$ Sets of DGs connected at bus $n$.

$\mathcal{B}_{n} \quad$ Sets of batteries connected at bus $n$.

$\mathcal{H}_{n} \quad$ Sets of houses connected at bus $n$.

$\mathcal{V}_{n} \quad$ Sets of PV connected at bus $n$.

$\Omega \quad$ Sets of feeders.

Variables

Binary Variables

$u_{i t} \quad 1$ if DG $i$ is scheduled on during period $t$ and 0 otherwise.

$u_{h t^{\prime}}^{\mathrm{C}} u_{h t}^{\mathrm{H}} \quad 1$ if HVAC of house $h$ is scheduled cooling/heating during period $t$ and 0 otherwise.

$u_{b t}^{\mathrm{C}}, u_{b t}^{\mathrm{D}} \quad 1$ if battery $b$ is scheduled charging/discharging during period $t$ and 0 otherwise.

Continuous Variables

$p_{i t}(m) \quad$ Power output scheduled from the $m$-th block of energy offer by DG $i$ during period $t$. Limited to $p_{i t}^{\max }(m)$.

$P_{i t}, Q_{i t} \quad$ Real/reactive power output scheduled from DG $i$ during period $t$.

$P_{b t}^{\mathrm{C}}, P_{b t}^{\mathrm{D}} \quad$ Charging/discharging power of battery $b$ during period $t$.

$Q_{b t} \quad$ Reactive power of battery $b$ during period $t$.

$S O C_{b t} \quad$ State of charge of battery $b$ during period $t$.

$P_{t}^{\mathrm{PCC}}, Q_{t}^{\mathrm{PCC}}$ Real/ reactive power at $\mathrm{PCC}$ during period $t$.

$P_{h t}, Q_{h t} \quad$ Real/reactive load of house $h$ during period $t$.

$P_{h t}^{\mathrm{LS}}, Q_{h t}^{\mathrm{LS}} \quad$ Real/reactive load curtailment of house $h$ during period $t$.

$T_{h t}^{\mathrm{In}} \quad$ Indoor temperature of house $h$ during period $t$.

$T_{h t}^{\mathrm{M}} \quad$ The temperature of thermal accumlating layer of inner walls and floor in house $h$ during period $t$.

$T_{h t}^{\mathrm{E}} \quad$ The temperature of house envelop during period $t$.

$V_{n t} \quad$ Voltage magnitude of bus $n$ during period $t$.

$P_{f t}, Q_{f t} \quad$ Real/ reactive power flow on line $f$ during period $t$.

$l_{f t} \quad$ Squared amplitudes of current on line $f$ during period $t$.

\section{Constants}

$\lambda_{i t}(m) \quad$ Marginal cost of the $m$-th block of energy offer by DG $i$ during period $t$.

$\lambda_{t}^{\mathrm{PCC}} \quad$ Purchasing price of energy from distribution grid during period $t$.

$C_{b t} \quad$ Degradation cost of battery $b$ during period $t$.

$V_{h t}^{\mathrm{LS}} \quad$ Cost of load curtailment of house $h$ during period $t$.

$P_{i}^{\max }, P_{i}^{\min }$ Maximum/minimum output of DG $i$.

$P^{\text {PCC,max }}$ Maximum input/output power at PCC.

$P_{v t}, Q_{v t} \quad$ Real/reactive power output from PV $v$ during period $t$.

$P_{h t}^{\mathrm{O}}, Q_{h t}^{\mathrm{O}} \quad$ Real/reactive power consumption scheduled for non-HVAC load in house $h$ during period $t$.

$P_{h}^{H} \quad$ Rated power of HVAC system in house $h$.

$P_{b}^{\mathrm{C}, \max }, P_{b}^{\mathrm{D}, \max }$ Maximum charging/discharging power of battery $b$.

$S O C_{b t}^{\max }, S O C_{b t}^{\min }$ Maximum/minimum state of charge of battery $b$ during period $t$.

$P_{h t}^{\mathrm{LS}, \max } \quad$ Maximum load curtailment of house $h$ during period $t$.

$\eta_{b}^{\mathrm{C}}, \eta_{b}^{D} \quad$ Battery charging/discharging efficiency.

$\omega_{h t} \quad$ Customer discomfort cost of house $h$ during period $t$.

$\delta_{h t} \quad$ Allowed temperature deviation of house $h$ during period $t$.

$\kappa_{i} \quad$ Operating Cost of DG $i$ at the point of $P_{i}^{\min }$.

$T_{t}^{\mathrm{A}} \quad$ Ambient temperature during period $t$. 
$T_{h t}^{\mathrm{D}} \quad$ Desired indoor temperature of house $h$ during period $t$.

$\Phi_{t} \quad$ Solar irradiance during period $t$.

$R_{h}^{\mathrm{A}} \quad$ Thermal resistance between room air and ambient of house $h$.

$R_{h}^{\mathrm{M}} \quad$ Thermal resistance between room air and the thermal accumulating layer in the inner walls and floor of house $h$.

$R_{h}^{\mathrm{E}} \quad$ Thermal resistance between room air and the envelope of house $h$.

$R_{h}^{\mathrm{EA}} \quad$ Thermal resistance between the envelope of house $h$ and the ambient.

$C_{h}^{\ln } \quad$ Total thermal capacitance of the indoor air of house $h$.

$C_{h}^{\mathrm{M}} \quad$ Total thermal capacitance of the inner walls of house $h$.

$C_{h}^{\mathrm{E}} \quad$ Total thermal capacitance of the house envelope of house $h$.

$p_{h}^{s} \quad$ The fraction of solar radiation entering the inner walls and floor of house $h$, then the rest of the solar energy is absorbed by the indoor air.

$\eta_{h} \quad$ Coefficient of performance (COP) of HVAC in house $h$.

$V_{\mathrm{thr}}^{\max }, V_{\mathrm{thr}}^{\min }$ Maximum/Minimum voltage thresholds beyond which voltage deviation will be minimized.

$V^{\max }, V^{\min }$ Maximum/Minimum voltage deviations.

$S_{i}, S_{b}, S_{f} \quad$ Apparent power limit of DG $i$, battery $b$ and line $f$.

$r_{f}, x_{f} \quad$ resistance and reactance of line $f$.

$I_{f}^{\max } \quad$ Maximum amplitudes of current on line $f$.

$\tan \left(\theta_{i}\right) \quad$ Power factor limit of DG $i$.

$\tan \left(\theta_{b}^{\mathrm{C}}\right), \tan \left(\theta_{b}^{\mathrm{D}}\right)$ Power factor limits of battery $b$ when charging/discharging.

$\tan \left(\theta^{\text {PCC, max }}\right)$ Power factor limits of PCC.

$\tan \left(\varphi_{h}^{\mathrm{H}}\right), \tan \left(\varphi_{h}^{\mathrm{O}}\right)$ Power factor of HVAC and non-HVAC load at house $h$.

\section{Bibliography}

1. Lasseter, R.; Akhil, A.; Marnay, C.; Stephens, J; Dagle, J.; Guttromson, R.; Meliopoulous, A.S.; Yinger, R.; Eto, J. CERTS Microgrid Concept, April 2002. Available online: https://pserc.wisc.edu/documents/research_documents/certs_documents/certs_publications / certs_micro grid/certsmicrogridwhitepaper.pdf (accessed 15, August 2017).

2. Madureira A.G.; Pecas Lopes, J.A. Coordinated voltage support in distribution networks with distributed generation and microgrids. IET Renew. Power Gen. 2009, 3, 439-454.

3. Beer, S.; Gomez, T.; Dallinger, D.; Momber, I,; Marnay, C.; Stadler, C. An economic analysis of used electric vehicle batteries integrated into commercial building microgrids. IEEE Trans. Smart Grid 2012, 3, 517-525.

4. Tsikalakis, A.G.; Hatziargyriou, N.D. Centralized control for optimizing microgrids operation. IEEE Trans. Energy Convers. 2008, 23, 241-248.

5. Agrawal, M.; Mittal, A. Microgrid technological activities across the globe: A review. Int. J. Res. Rev. Appl. Sci. 2011, 7, 147-152.

6. Gu, W.; Wu, Z.; Bo, R.; Liu, W.; Zhou, G.; Chen, W.; Wu, Z.; Modeling, planning and optimal energy management of combined cooling, heating and power microgrid: A review. Int. J. Electr. Power Energy Syst. 2014, 54, 26-37.

7. Belvedere, B.; Bianchi, M.; Borghetti, A.; Nucci, C.A.; Paolone, M.; Peretto, A. A microcontroller-based power management system for standalone microgrids with hybrid power supply. IEEE Trans. Sustain. Energy 2012, 3, 422-431.

8. Morais, H.; Kadar, P.; Faria, P.; Vale, Z.A.; Khodr, H.M. Optimal scheduling of a renewable micro-grid in an isolated load area using mixed-integer linear programming. Renew. Energy 2010, 35, 151-156.

9. Babazadeh, H.; Gao, W.; Wu, Z.; Li, Y. Optimal energy management of wind power generation system in islanded microgrid system. In Proceedings of the North American Power Symposium(NAPS), Manhattan, Kansas, USA, 22-24 September 2013.

10. Palma-Behnke, R.; Benavides, C.; Lanas F.; Severino, B.; Reyes, L.; Llanos, J.; Saez, D. A microgrid energy management system based on the rolling horizon strategy. IEEE Trans. Smart Grid 2013, 4, 996-1006.

11. Sobu, A.; Wu, G. Dynamic optimal schedule management method for microgrid system considering forecast errors of renewable power generations. In Proceedings of the 2012 IEEE International Conference on Power System Technology(POWERCON), Auckland, New Zealand, 30 October-2 November 2012. 
12. Mohamed, F.A.; Koivo, H.N. System modelling and online optimal management of MicroGrid using Mesh Adaptive Direct Search. Int. J. Electr. Power Energy Syst. 2010, 32, 398-407.

13. Cardoso, G.; Stadler, M.; Siddiqui, A.; Marnay, C,; DeFrost, N.; Barbosa-Povoa, A.; Ferrao, P. Microgrid reliability modeling and battery scheduling using stochastic linear programming. Electr. Power Syst. Res. 2013, 103, 61-69.

14. Nguyen, D.T.; Le, L.B. Optimal Bidding Strategy for Microgrids Considering Renewable Energy and Building Thermal Dynamics. IEEE Trans. Smart Grid 2014, 5, 1608-1620.

15. Liu, G.; Xu, Y.; Tomsovic, K. Bidding Strategy for Microgrid in Day-Ahead Market Based on Hybrid Stochastic/Robust Optimization. IEEE Trans. Smart Grid 2016, 7, 227-237.

16. Daratha, N.; Das, B.; Sharma, J. Coordination Between OLTC and SVC for Voltage Regulation in Unbalanced Distribution System Distributed Generation. IEEE Trans. Power Syst. 2014, 29, 289-299.

17. Dolan, M.J.; Davidson, E.M.; Kockar, I.; Ault, G.W.; McArthur, S. Distribution Power Flow Management Utilizing an Online Optimal Power Flow Technique. IEEE Trans. Power Syst. 2012, 27, 790-799.

18. Paudyal, S.; Canizares, C.A.; Bhattacharya, K. Optimal Operation of Distribution Feeders in Smart Grids. IEEE Trans. Ind. Electron. 2011, 58, 4495-4503.

19. Borghetti, A.; Bosetti, M.; Grillo, S.; Massucco, S.; Nucci, C.; Paolone, M.; Silvestro, F. Short-term scheduling and control of active distribution systems with high penetration of renewable resources. IEEE Syst. J. 2010, 4, 313-322.

20. Zhou, Q.; Bialek, J. Generation curtailment to manage voltage constraints in distribution networks. IET Gener., Transm., Distrib. 2007, 1, 492-498.

21. Calderaro, V.; Conio, G.; Galdi, V,; Massa, G.; Piccolo, A. Optimal Decentralized Voltage Control for Distribution Systems With Inverter-Based Distributed Generators. IEEE Trans. Power Syst. 2014, 29, $230-241$.

22. Liu, G.; Ceylan, O.; Xu, Y.; Tomsovic, K. Optimal Voltage Regulation for Unbalanced Distribution Networks Considering Distributed Energy Resources. In Proceedings of the IEEE Power \& Energy Society General Meeting (PESGM), Denver, Colorado, USA, 26-30 July 2015

23. Liu, G.; Xu, Y.; Ceylan, O.; Tomsovic, K. A new linearization method of unbalanced electrical distribution networks. In Proceedings of the North American Power Symposium(NAPS), Pullman, Washington, USA, 7-9 September 2014.

24. Shekari, T.; Golshannavaz, S,; Aminifar, F. Techno-Economic Collaboration of PEV Fleets in Energy Management of Microgrids. IEEE Trans. Power Syst. 2017, 32, 3833-3841.

25. Trakas, D.; Hatziargyriou, N. Optimal Distribution System Operation for Enhancing Resilience Against Wildfires IEEE Trans. Power Syst. 2017, 99, 1-1.

26. Lavaei, J.; Low, S.H. Zero Duality Gap in Optimal Power Flow Problem. IEEE Trans. Power Syst. 2012, 27, 92-107.

27. Jabr, R. Radial distribution load flow using conic programming. IEEE Trans. Power Syst. 2006, 21, 1458-1459.

28. Baran, M.; Wu, F. Optimal capacitor placement on radial distribution systems. IEEE Trans. Power Syst. 1989, $4,725-734$.

29. Farivar, M.; Low, S. Branch flow model: Relaxations and convexification -Part I. IEEE Trans. Power Syst. 2013, 28, 2554-2564.

30. Nguyen D.T.; Le, L.B. Joint optimization of electric vehicle and home energy scheduling considering user comfort preference. IEEE Trans. Smart Grid 2014, 5, 188-199.

31. Nguyen D.T.; Le, L.B. Optimal Bidding Strategy for Microgrids Considering Renewable Energy and Building Thermal Dynamics. IEEE Trans. Smart Grid 2014, 5, 1608-1620.

32. Saaty, T. Decision making-The analytic hierarchy and network processes (AHP/Anp). J. Syst. Sci. Syst. Eng. 2004, 13, 1-35.

33. Thavlov, A. Dynamic optimization of power consumption. M.S. thesis, Tech. Univ. Denmark, Kongens Lyngby, Denmark, 2008.

34. Madsen, H.; Holst, J. Estimation of continuous-time models for the heat dynamics of a building. Energy Buildings 1995, 22, 67-79.

35. Bacher, P.; Madsen, H. Identifying suitable models for the heat dynamics of buildings. Energy Buildings 2011, 43, 1511-1522.

36. Siroky, J.; Oldewurtel, F.; Cigler, J,; Privara, S. Experimental analysis of model predictive control for an energy efficient building heating system. Appl. Energy 2011, 88, 3079-3087. 
37. Taylor, J.A.; Hover, F.S. Conic AC transmission system planning. IEEE Trans. Power Syst. 2013, 28, 952-959.

38. Baradar, M.; Hesamzadeh, M.R.; Ghandhari, M. Second-order cone programming for optimal power flow in vsc-type ac-dc grids. IEEE Trans. Power Syst. 2013, 28, 4282-4291.

39. Carrion, M.; Arroyo, J.M. A computationally efficient mixed-integer linear formulation for the thermal unit commitment problem. IEEE Trans. Power Syst. 2006, 21, 1371-1378.

40. Oak Ridge National Laboratory (ORNL) Rotating Shadowband Radiometer (RSR). Available online: https:/ / www.nrel.gov/midc/ornl_rsr/ (accessed 15, August 2017). 